Geological Society, London, Special Publications, 122, 1-10, 1 January 1997, https://doi.org/10.1144/GSL.SP.1997.122.

\title{
A non-contacting resistivity imaging method for characterizing whole round core while in its liner
}

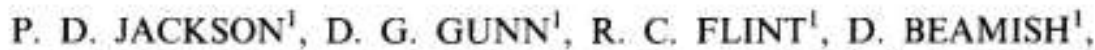 \\ P. I. MELDRUM ${ }^{1}$, M. A. LOVELL ${ }^{2}$, P. K. HARVEY ${ }^{2}$ \& A. PEYTON ${ }^{3}$ \\ ${ }^{1}$ British Geological Survey, Keyworth Nottingham, NG12 5GG, UK \\ ${ }^{2}$ University of Leicester, University Road, Leicester LEI 7RH, UK \\ ${ }^{3}$ UMIST, Manchester M60 IQD, UK
}

\begin{abstract}
Recent laboratory experimentation has shown that non-contacting whole-core resistivity imaging, with azimuthal discrimination, is feasible. It has shown the need for very sensitive coil pairs in order to provide resistivity measurements at the desired resolution. Independent high-resolution 'galvanic' resistivity estimations show the 'non-contacting' measurements to be directly proportional to the resistivity of core samples. The response of the technique to a variety of synthetic 'structures' is presented. A whole-core image of a dipping layer is used to demonstrate the three dimensional response of the technique and to show that the resolution of the measurements is of the order of $10 \mathrm{~mm}$.

Experiments are described which show that the technique is capable of investigating to different depths within the whole round core. The results agree with theoretical predictions and indicate that the technique has the potential to assess invasion near the surface of the core.

The technique is intrinsically safe and has the potential to be packaged in a form that would be suitable for whole-core imaging at the well site, or laboratory, without taking core from their liners. Thus it is possible to acquire information crucial for core selection, in addition to acquiring resistivity data at a resolution not too far removed from that of the downhole imaging tools.
\end{abstract}

A review of research and development requirements in core analysis, sponsored by the Department of Trade and Industry Oil and Gas and Supplies Office (HMSO 1991), identified the potential of contacting resistivity imaging (Jackson et al. 1995; Lovell et al. 1995) but concluded that its widespread, routine, application was likely to be held back by the need to make direct electrical contact with the core. Non-contacting resistivity imaging was seen in the review to offer the possibility of portable, non-radiation-dependent scanning technology for use offshore. The primary purpose of such technology being to provide calibration data for downhole electrical images, identify core for special analyses and to lead to an understanding of heterogeneities of direct relevance to other logging measurements at the scale of sedimentary features.

The objective of the research reported here was to demonstrate the feasibility of a nondestructive, non-contacting resistivity method, for continuously logging oil industry core.

Contacting resistivity measurements are routinely made by the oil industry on cylindrical sub-samples taken from whole-round core. Only a small fraction of the core is sampled in this way, additional samples at different orientations are required to assess anisotropy, "average core-plug' values are obtained which are not representative of small-scale sedimentary features and the process is destructive to the core.

Routine, continuous high-resolution, nondestructive, non-contacting resistivity logging of oil-field core would, for the first time, provide core-resistivity datasets comparable to the corresponding downhole ones, that could be integrated with both downhole logs and borehole wall images to aid scaling up borehole datasets for use in reservoir studies.

\section{Background}

Contacting resistivity imaging of core (Jackson et al. 1995) has provided high-resolution images of the electrical resistivity of the central portion of cores using electrodes adjacent to the slabsurface surface of core which had been bisected by a single saw cut parallel to its long axis. These techniques have resolutions of $5 \mathrm{~mm}$ and have been used to investigate the control of fabric, heterogeneity and planar sedimentary features on the flow of fluids through sandstone samples which are an analogue for the Rotleigendes Formation of the southern North Sea (Lovell et al. 1995; Harvey et al. 1995). 
While these techniques are suitable for specialist laboratory investigations on core plugs and slabbed core, they are not suitable for use on core as it arrives at the well-site or laboratory, due to the presence of mud and irregular surfaces, which can typify whole-round core. Non-contacting methods, while having lower resolution, could be used on the entire suite of core that is collected, before it is removed from its liners, providing a spatially complete dataset for comparison to that obtained downhole. The present practice of subsampling core-plugs for resistivity measurements, provides an extremely sparse and incomplete data seí.

Non-contacting electromagnetic methods are common in geophysical prospecting, but often use perturbations of the primary magnetic field to assess geological structure. These measurements are not directly related to the Earth's electrical resistivity. However, the currents induced in the Earth during such measurements are controlled by the resistivity of the material in which they flow and give rise to a secondary magnetic field which is directly proportional to the induced current. Therefore the secondary magnetic field, typically extremely small, is directly related to the electrical resistivity of the formation.

Although non-contacting resistivity induction logging has been used by the oil industry for almost 50 years (Doll 1949; Moran \& Kuntz 1962 ), it is not used extensively for measurements on core material.

Gerland et al. (1993) used a modified instrument designed originally for assessing the electrical conductivity of small-diameter, cylindrical, ore-bearing hard-rock to assess the resistivity of marine sediments. They passed cylindrical core through a hole in the centre of a single coil. The arrangement produced resistivity values that appear to be averaged over $100 \mathrm{~mm}$ length of whole-core, sharp transitions in resistivity being smeared out over $100 \mathrm{~mm}$.

Huang et al. (1993, 1995) describe the operation and use a system of coils which appear to have been scaled down from those first used for induction logging. They passed a coil array along the axis and through the centre of a special cylindrical sample holder, that contained the loose material under investigation.

The two approaches described above are suitable for imaging sedimentary structures within core because they respond to the whole of the core or sample over a depth interval of perhaps $100 \mathrm{~mm}$, and have no azimuthal discrimination.

\section{Non-contacting resistivity imaging of core}

In order to overcome these problems, and to investigate azimuthally variable sedimentalogical structure within whole-round core, we investigated an approach based on scaling down conventional, electromagnetic prospecting which has been used successfully in engineering and environmental studies.

Electromagnetic geophysical surveying has traditionally used two coil systems, of one transmitter and one receiver, (e.g. Keller \& Frischknecht 1966; Telford et al. 1976). The objective of such surveys is to identify the magnetic field at the receiver coil, which itself is a combination of the magnetic field produced by the transmitter and the magnetic field that arose due to currents induced in the Earth by the primary magnetic field, the primary field being created by current flowing in the transmitter coil.

At low frequencies, resistive terms dominate the governing equations and the secondary currents and their magnetic field are about $90^{\circ}$ out of phase with the primary field. At very high frequencies skin effects dominate and the secondary fields can be $180^{\circ}$ out of phase with the primary field. At low frequencies the induced currents can flow deeply within the Earth, whereas at high frequencies they flow near the surface (i.e, skin effect) and can, under limiting conditions, cancel the primary field. This latter effect has been referred to as "eddy current saturation' and is most likely in the presence of highly conductive material.

The value of a 'low' or a 'high' frequency depends on the electrical conductivity (1/resistivity) of the material under investigation and the dimension of the problem in terms of the separation of the transmitting and receiving coils.

The low-frequency limit in electromagnetic geophysical surveying has become popular when the low induction-number criterion is also met (McNeil 1979). In this limit, the skin depth is far greater than the coil separation and the mutual induction of the secondary currents can be neglected. The technique has now become the method of choice for rapid, reconnaissance-style resistivity surveys for engineering and environmental studies.

Measurements of the secondary magnetic field, at low induction number, provide values that are inversely proportional to the electrical resistivity of the material being investigated. As such measurements could be made with small coil pairs at the surface of cylindrical rock cores, this technique was selected to be developed for non-contacting imaging of 'whole-round' core. 
For a horizontally layered Earth, in the low induction number approximation, each 'filament' of induced current is considered to be independent of the other induced currents. This current flow is controlled by the primary field and the electrical conductance of the 'filament' in which it is flowing, and is unaffected by the electrical conductivity of the surrounding material. Response curves can be calculated, showing the relative contribution to the secondary field of different layers, as shown in Fig. 1. These curves enable the coil spacing and coil coupling to be optimised for the depth range of interest and for the theoretical response to be calculated. It is also possible to undertake localized electromagnetic 'soundings' using coil arrangements with gradually increasing depths of investigation, which can be interpreted in terms of layer thickness and resistivities. Figure $\mid$ also demonstrates very different responses to near surface layers when the dipoles are changed from a vertical to a horizontal orientation.

For the laboratory situation where imaging sedimentalogical structures within cylindrical cores is required, a reasonable premise is that the dimensions of the transducer coils will be a compromise between very small, to identify sedimentalogical structures, and being large enough to ensure the secondary fields are measurable to the desired degree of accuracy.

\section{Design of sensor coils}

As described above, for non-contacting, lowinduction-number, resistivity measurements, the primary magnetic field produced by the transmitter coil induces electric current in the core. These currents, in turn, generate a secondary magnetic field, which is inversely proportional to the resistivity of the core. The measurement of this secondary field is complicated because it is typically 107 smaller than the primary one. In order to measure the secondary field accurately we found it necessary to remove the primary field and amplify the residual field (secondary) to measurable levels. This is a complicated procedure, as at each stage it is necessary to avoid degradation of the minute secondary signal, while at the same time maximising its amplitude.

The measurement sensors are inductive coils. Experimentation showed the need for these coils to be specially designed, in order to exclude extraneous signals and to optimise the secondary signal. Sensor coils were designed and developed to be sensitive to changes in resistivity occurring over lateral distances of $10-20 \mathrm{~mm}$ over the cylindrical surface of whole-core. The coils were fixed at a separation of $\mathbf{4 2} \mathrm{mm}$ for the experiments described below. In the first instance, the coil separation was selected to

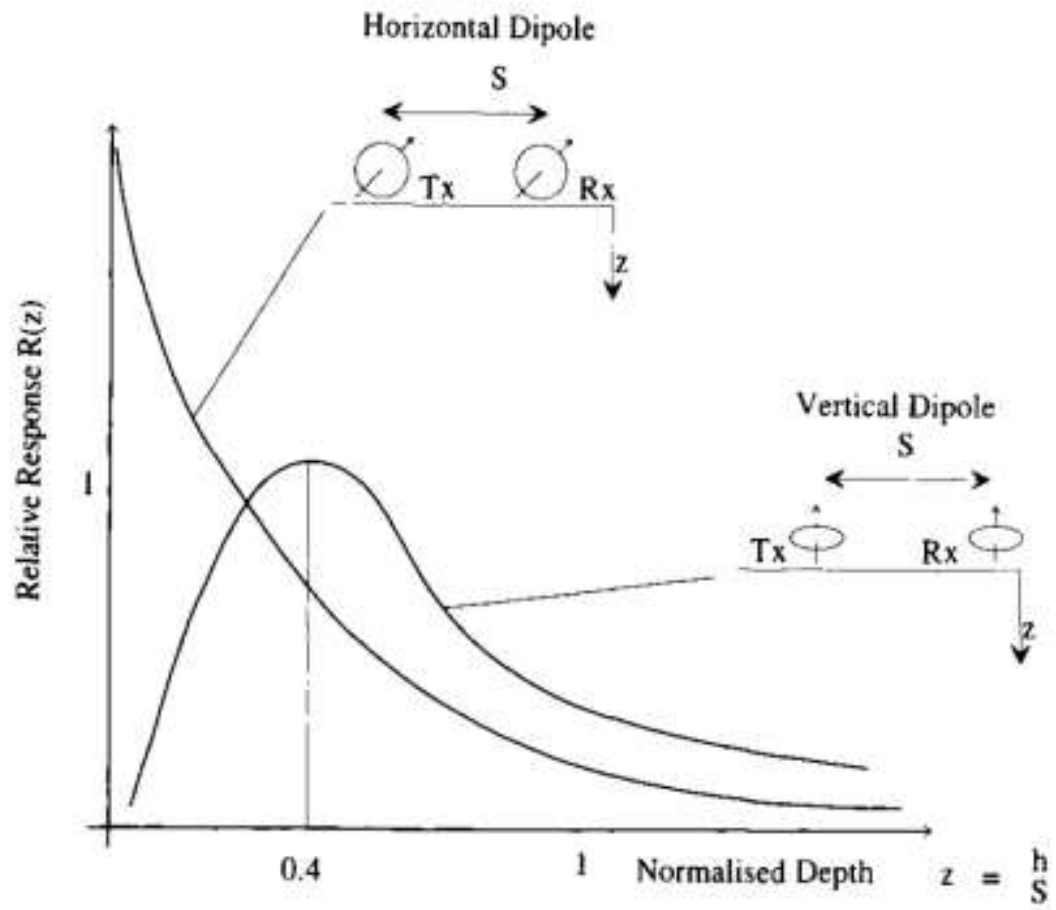

Fig. 1. Theoretical responses for horizontal and vertical dipole arrangements. 
provide a substantial depth of investigation into the core to ensure edge effects did not dominate the performance. Consequently there is substantial potential for increasing the resolution by reducing the coil separation.

It is essential that the measurement provides an accurate and quantitative assessment of the resistivity of the core. In order to confirm the performance of the technology, measurements were made on the surface of a cylindrical Plexiglas tube, of internal diameter $100 \mathrm{~mm}$, filled with a fluid of known electrical resistivity. Figure 2 demonstrates the performance of both
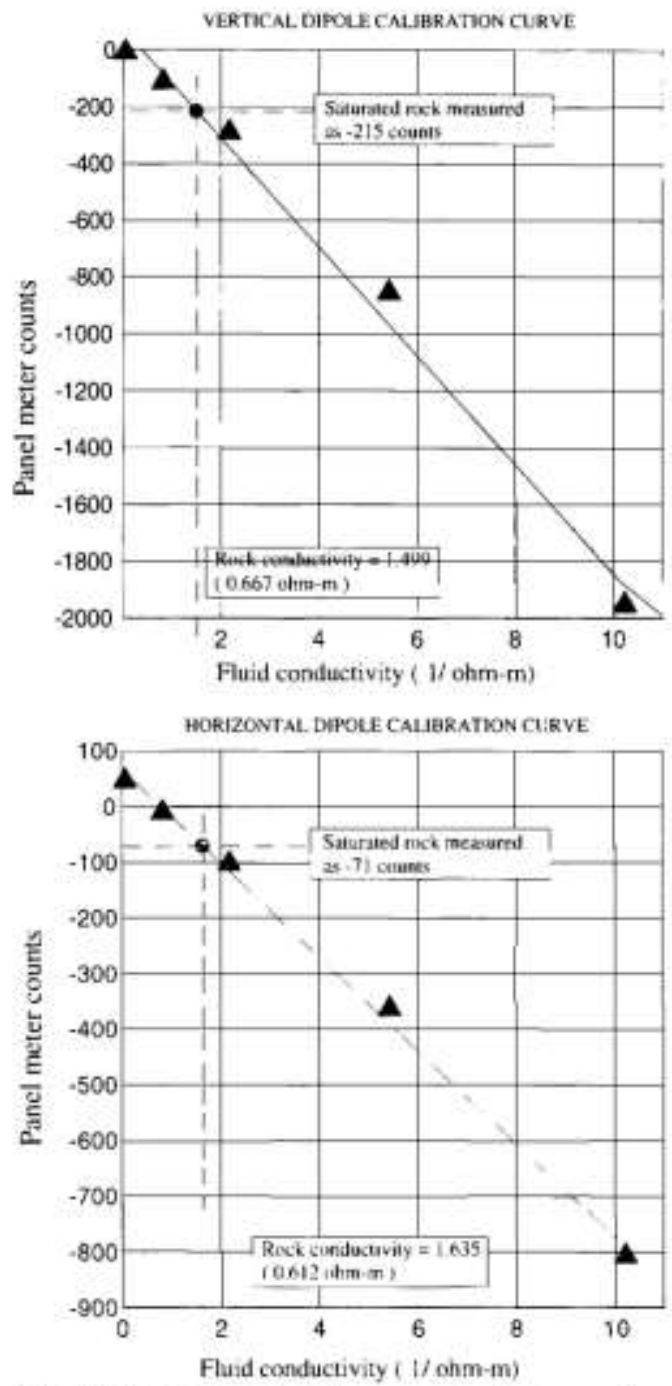

Fig. 2. Resistivity calibration curves for horizontal and vertical dipole arrangements.

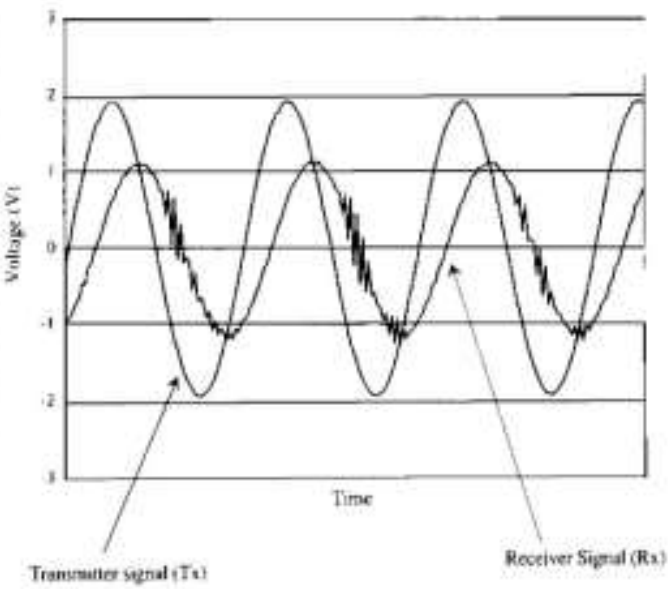

Fig. 3. Transmitter and receiver signals showing a $90^{\circ}$ phase shift after processing to remove the primary magnetic field. The receiver signal is amplified relative to the transmitter signal.

horizontal and vertical dipoles using fluids having resistivities in the range $0.25-10 \Omega \mathrm{m}$. The responses can be seen to be directly proportional to the electrical conductivity of fluid inside the core tube, confirming the measurement system is performing as expected. These data exhibit a straight line relationship between the measurement of the secondary field and the fluid conductivity, and were used to calibrate the output in terms of the resistivity of cylindrical core. Examples of the signals seen at the Transmitter $(\mathrm{Tx})$ coil (primary field) and the Receiver ( $R x)$ coil (secondary field) are shown in Fig. 3, illustrating the $90^{\circ}$ phase difference between them. The $\mathrm{Rx}$ signal has been processed to remove the primary field generated by the $\mathrm{Tx}$, and can be seen to have the greater noise. This is consistent with the Rx signal being extremely small compared to the Tx signal.

\section{Experimental results}

The performance of the sensor system to bed boundaries was studied using insulating 'targets' placed within the Plexiglas, fluid-filled, cylindrical tube described above. The results shown in Fig. 4 demonstrate that bed boundaries corresponding to changes in rock resistivity can be resolved. This was seen to be a crucial initial step towards the development of an imaging capability. Figure 4 shows the coil response to three horizontal resistive layers of differing thickness. The results resolve the interfaces of the layers 

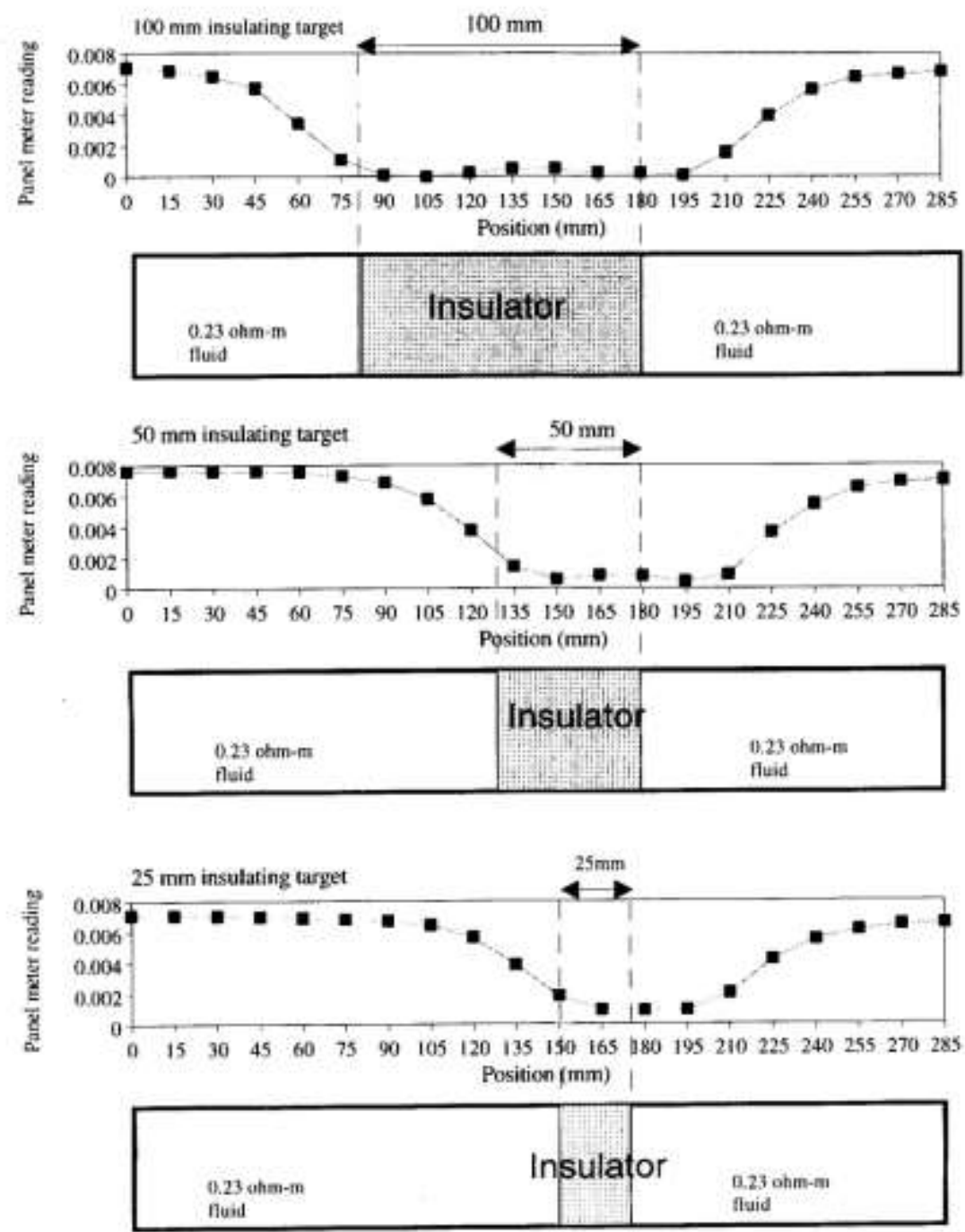

Fig. 4. Response to horizontal resistive layers of differing thicknesses. Dotted lines show where the leading and trailing edges pass the first $(\mathbf{R x})$ coil.

even when the layer thickness is less than the coil separation. Note the results are plotted as a log along the length of the core.

Multiple data sets were acquired to demonstrate the use of non-contacting resistivity methods in producing resistivity images. Figure 5 demonstrates the application of the technology in image acquisition mode. In order to build up an image, the cylindrical sample was measured at $15 \mathrm{~mm}$ intervals along its length and at 12 azimuthal positions around its circumference for each horizontal move. The data relate to a slab of low-porosity rock within a conductive fluid.
Figure 5 shows the coil response to a resistive layer with one horizontal interface and one dipping interface. The data are presented as an unrolled image, in addition to individual logs corresponding to maximum and minimum thickness (long core axis and short core axis), with the dark areas representing low conductivity (high resistivity) and the light areas representing high conductivity (low resistivity).

A comparison of Figs 4 and 5 shows that while the thickness of each layer is overestimated by a distance approximately equal to the coil separation, the dipping interface in Fig. 5 
Schematic diagram of tapered rock in fluid filled tube.

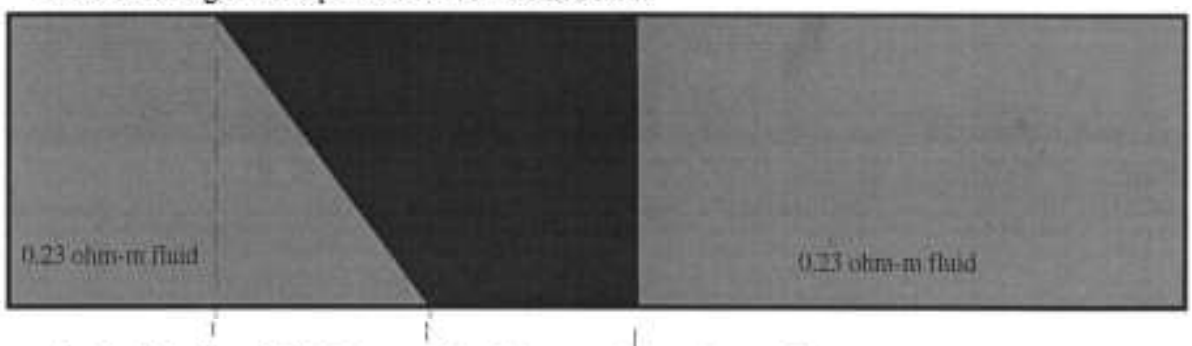

Conductivity log with the longest side of the core adjacent the to coils.

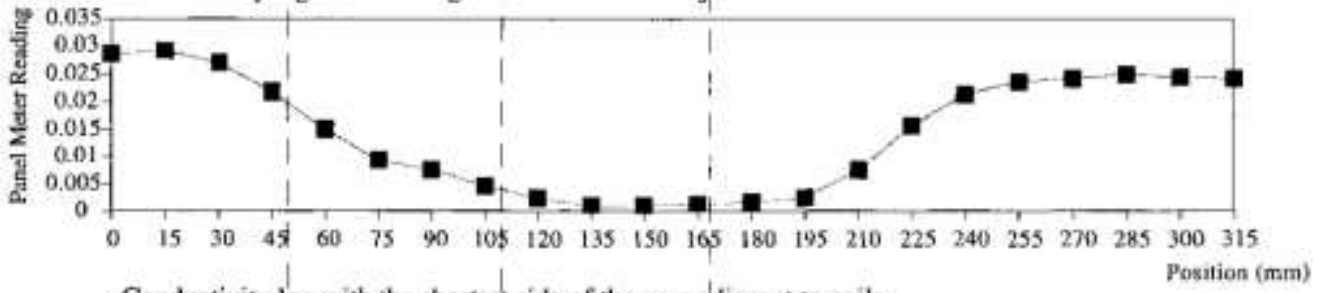

Conductivity ipg with the shortest side of the core adjacent to conls.

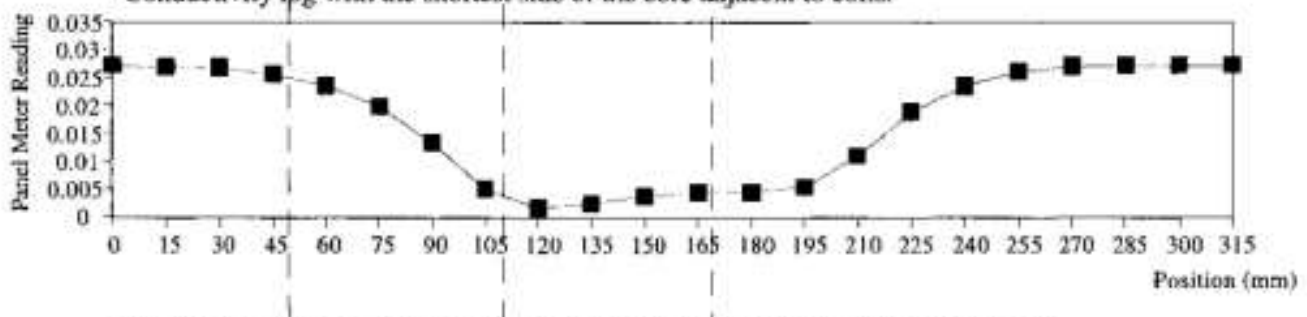

Image of complete sample from Conm to $285 \mathrm{~mm}$ utilising twelve 30 degree motations.

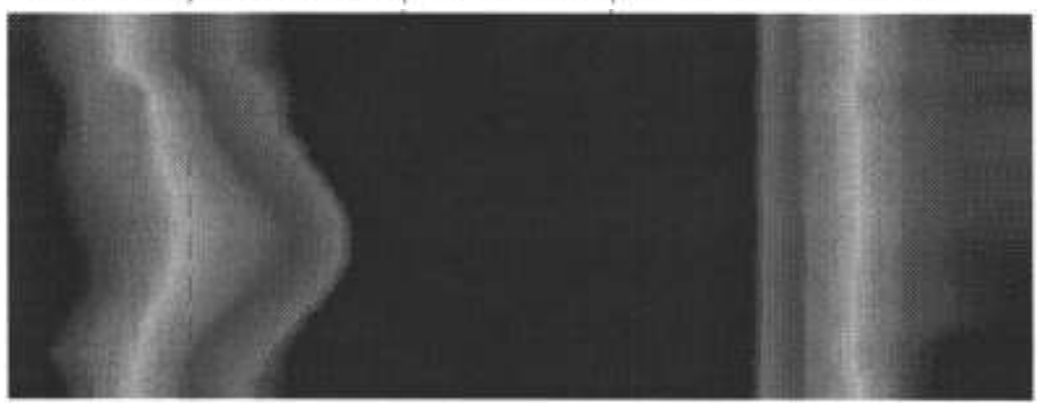

Fig. 5. Response to a resistive layer with one horizontal and one dipping interface. Dotted lines show where the leading and trailing edges pass the first ( $\mathrm{Rx})$ coil.

has been spatially, faithfully reproduced as a sinusoid in the unrolled image. This latter observation suggests the lateral resolution and depth

of investigation of the coil array are suitable for imaging sedimentalogical structure within whole-round core, even in the presence of invasion of the core by drilling mud and filtrate, because the dipping structure has been identified even though a relatively large depth of investigation had been employed (comparable to the coil separation of $42 \mathrm{~mm}$ ).

This approach was extended to a dipping layer far thinner than the dimensions of the coil array. The experimental set up can be seen in Fig. 6 where the thin dipping layer is constructed from two sandsione cores, each with complimentary, parallel dipping-interfaces. The separation of the two dipping interfaces was fixed at $25 \mathrm{~mm}$, simulating a thin dipping conductive 


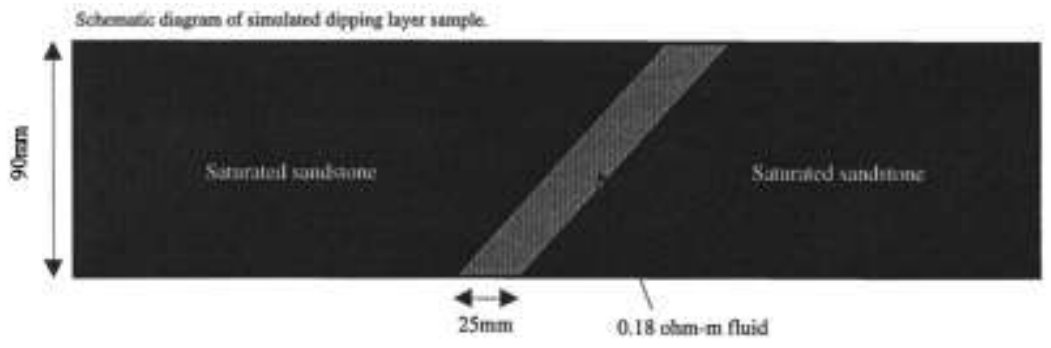

Confluctivity image of simmlated dipping linger sanple.

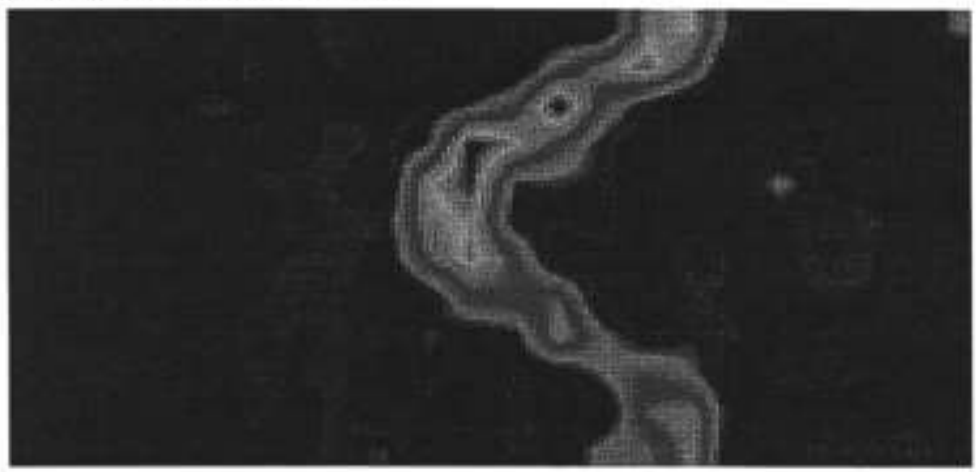

Fig. 6. Response to a dipping conductive layer.

layer, which could, for example, represent a narrow fluid-filled fracture. The resulting image is shown in Fig. 6 (bottom) with the dark areas representing low conductivity (high resistivity) with the converse applying to the light areas. The thin conducting layer is clearly discernible.

The use of different coil orientations was investigated as a means of assessing the resistivity structure of the core in three dimensions. This is of great importance because it would enable the assessment of, and compensation for, the effects of invasion on the core sample. A simple test of the response of the coil system in different orientations was carried out to demonstrate different depths of investigation that could be achieved.

Figure 7 shows the coil response for vertical and horizontal dipoles around a heterogeneous core. A resistive horizontal layer contains an azimuthal section of conductive material. This model is used to investigate the use of horizontal and vertical coil orientations in sampling different depths into the core. On the resistivityposition plot at the cut face both coil systems appear to be equally influenced by the near-field conductive fluid. In comparison, for the uncut face the vertical dipole continues to be influenced by the conductive fluid on the far side of the core whilst the horizontal dipole measures an apparent resistivity which is closer to that of the core.

With reference to Fig. 8 it can be seen that the area beneath the relative response curves for the horizontal and vertical dipoles between 10 and $35 \mathrm{~mm}$ depth (conductive fluid) could be the same fraction of the corresponding areas beneath the response curves for depths greater than $10 \mathrm{~mm}$ (the height of the $\mathrm{Tx}$ and $\mathrm{Rx}$ coils above the fluid/rock). This provides an explanation of the equivalence of the resistivity values in Fig. 7, for the two dipole-orientations, when the coil array is adjacent to the cut face. It can also be seen in Fig. 8 that the response curve for the horizontal dipole decreases, in a fractional sense, far more quickly than the response curve for the vertical dipole. This is consistent with the horizontal dipole having a shallower depth of investigation than the vertical one, explaining the measurement response, described above, when the coil array is adjacent to the uncut face.

\section{Conclusions}

The results have demonstrated the potential of using different non-contacting inductive coil arrays to investigate the resistivity structure of 

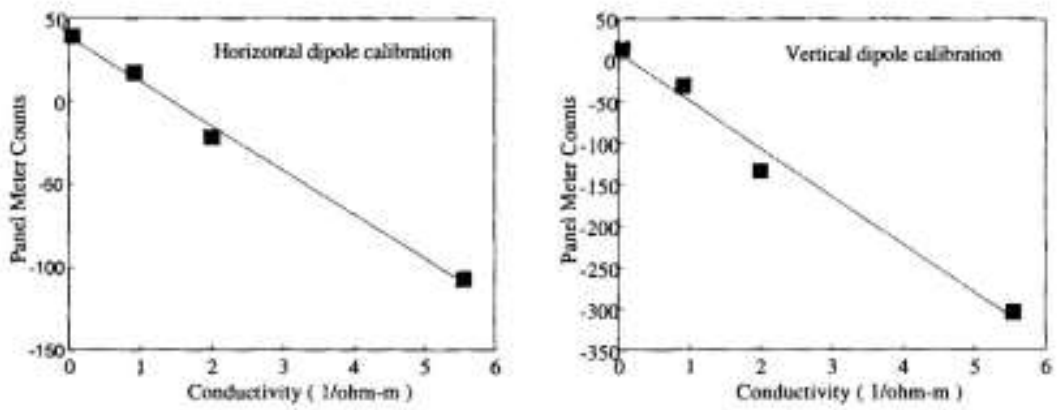

A plastic tube filled with Guids of four different resistivities was used for the above calibration.

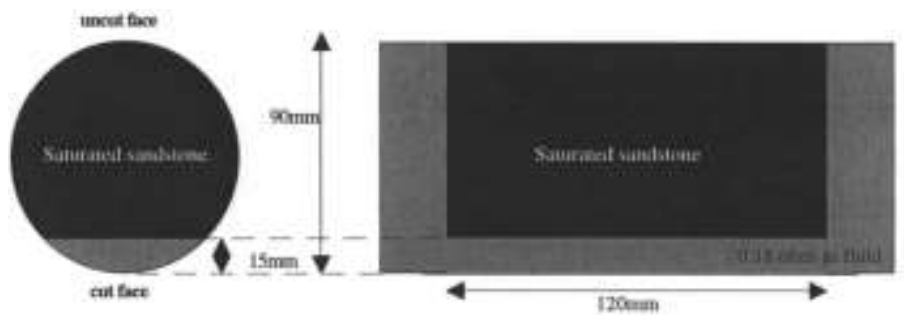

Log' Exasurensents co the soturated enre above for iwo depths of investigation at hoth fices.

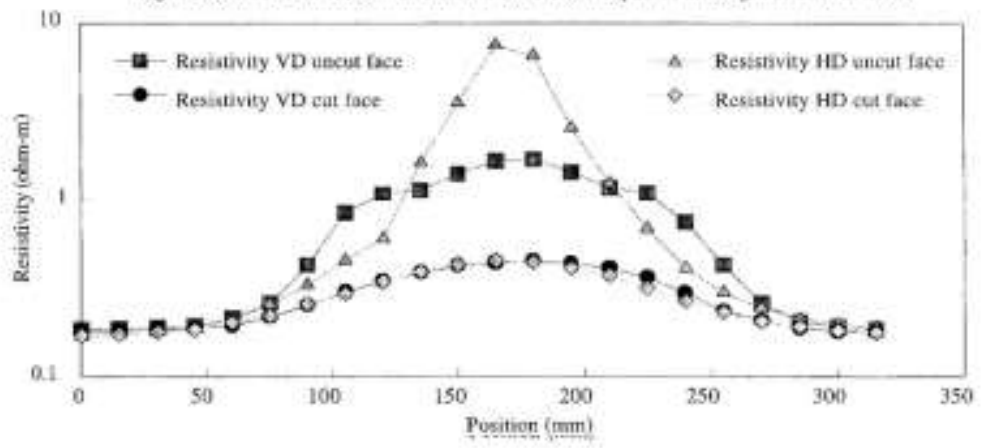

Fig. 7. Depth of investigation experiment using a resistive layer with a cut face.

whole-round core, It is readily apparent, however, that further, more detailed, investigation of the effects of coil separation, orientation and position would be beneficial. The results demonstrate the compatibility of our experimental investigations with theory and thus provide a basis for developing a full three dimensional capability.

The results provide an experimental basis to develop $2 \mathrm{D}$ and $3 \mathrm{D}$ portable, non-radiationdependent scanning technology for application to the bulk of the core retrieved during drilling operations, both offshore and in laboratories.
Resistivity assessments at a scale of $10-20 \mathrm{~mm}$ are anticipated, which will offer the possibility of whole-core characterization routinely to the industry for the first time. These data could be used to upscale core data to lower resolution, but deeper penetrating, resistivity logging data will, in addition, provide quantitative calibration of down hole conductance images of the borehole wall. This would lead to an understanding of heterogeneities of direct relevance to other logging measurements at the scale of significant sedimentary features which would aid scaling up data from the borehole to the reservoir scale. 


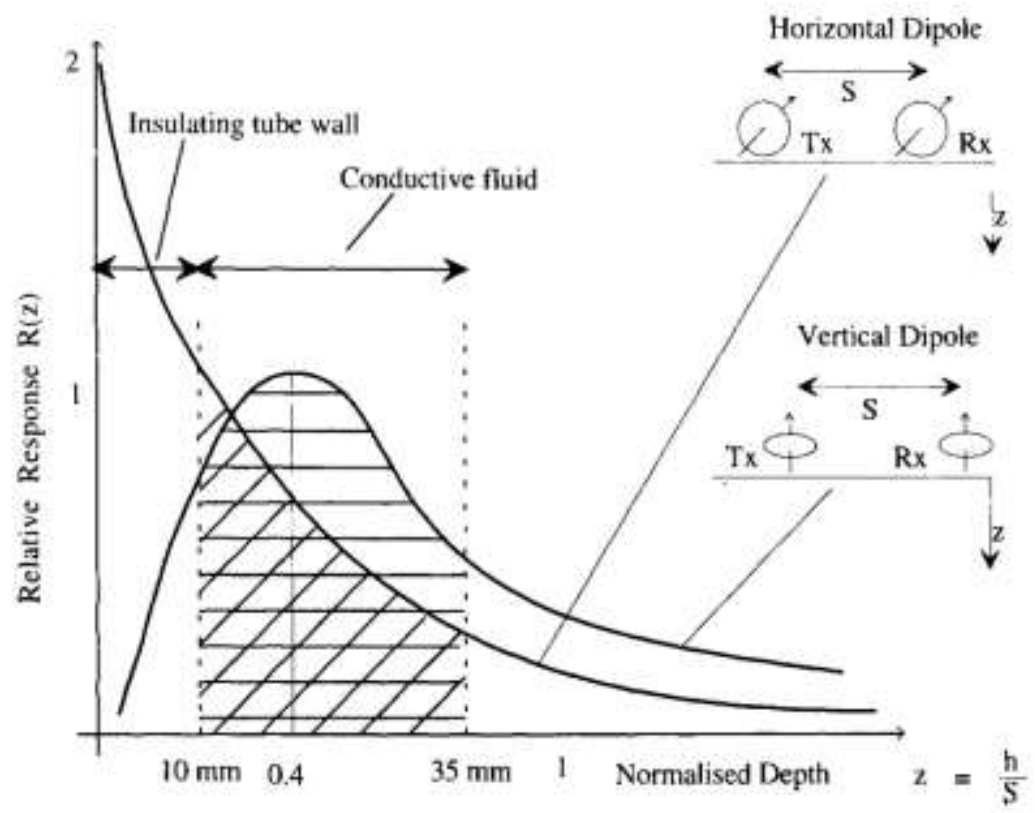

Fluid response for horizontal dipole

Fluid response for vertical dipole

Fig. 8. Response curves for horizontal and vertical dipoles showing the likely response of the fluid phase introduced by the cut face.

The research reported here was part supported by the Oil and Gas Projects and Supplies Office of the UK Department of Trade and Industry. This paper is published with the permission of the Director of the British Geological Survey (NERC).

\section{References}

DoLL, H. G. 1949. Introduction to induction logging and application to logging of wells drilled with oil base mud. Transactions of the American Institute of Mining and Metallurgical Engineers, 186, $148-162$.

HMSO 1991. Study of research and development requirements in core analysis, Geoscience report to the Department of Energy (OSO). Oet 1991 HMSO.

GERLAND, S., RichtER, M. VILLINGER, H. \& KUHN, G. 1993. Non-destructive porosity determination of Antaretic marine sediments derived from resistivity measurements with an inductive method. Marine Geophysical Researches, 15, 201-218.
Harvey, P. K., Lovell, M. A., Jackson, P. D., Ashu, A: P., WiLliamson, G., SMITH, A. S., BALL, J. K. \& FLINT, R. C. 1995. Electrical Resistivity Core Imaging III: Characterisation of an Aeolian Sandstone. Scientific Drilling. 5, $164-176$.

Huang, M., Lul, C. \& Shen, L. C. 1993. Laboratory resistivity measurement using a contactless coiltype probe. SPWLA 34th Annual Logging Symposium, paper JJ, June 13-16 1993.

- - — \& SHATTUK, D. 1995. Monitoring soil contamination using a contactless conductivity probe. Geophysical Prospecting, 43, 759-778.

JACKSON, P. D., LOVEl.L. M. A., HARveY, P. K., BALL, J. K., WILLAMS, C, FLINT, R. C., GUNN, D. A., Ashu, A. P. \& MeldruM, P. I. 1995. Electrical Resistivity Core Imaging I: A new technology for high resolution investigation of petrophysical properties. Scientific Drilling, 5, 139-151.

Kel.LeR, G. V. \& FrischKNeCHt, F, C. 1966. Electrical methods in geophysical prospecting. Pergamon Press, Oxford. 
Lovell, M. A., Harvey, P. K., Jackson, P. D., Flint, R, C, Gunn, D. A. Williamson, G, Balt, J. K., Ashu, A. P. \& Williams, C. 1995. Electrical Resistivity Core Imaging II: investigation of fabric and fluid flow characteristics. Scientific Drilling, 5, 153-164.

MCNeIL, J. D. 1979. Interpretive aids for use with electromagnetic (non-contacting) ground resistivity mapping. Forty-First European Association of
Exploration Geophysicists meeting, Hamburg, June 1979.

MoRAN, J. H. \& KUNTz, K. S. 1962. Basic theory of induction logging and application to study two coil sondes. Geophysics, 27, 829-858.

TElford, W. M., Geldhart, L. P. SHerifF, R. E. \& KEYs, D. A. 1976. Applied Geophysics. Cambridge University Press, London. 